\title{
EQUITÀ SENZA EGUAGLIANZA: UN ROMPICAPO ARISTOTELICO
}

\author{
MARIO VEGETTI (*)
}

SunTO. - Nel libro V dell'Etica nicomachea Aristotele affronta, nell'ambito del discorso sulla "giustizia distributiva" (un sottosistema rispetto alla "giustizia in generale", o politica, che consiste sostanzialmente nel rispetto delle leggi), la questione dell'equa ripartizione di beni sociali quali cariche di comando, posizioni di prestigio, ricchezze pubbliche. Occorre evitare sia l'inganno democratico di una ripartizione perfettamente egualitaria, perché non corrisponde alle reali gerarchie sociali di ceto e di merito, sia l'avidità (pleonexia) che è propria dei regimi oligarchici: tanto l'egualitarismo quanto l'avidità sono forieri di conflitti sociali. Aristotele dunque propone un concetto di equità "proporzionale", una sorta di equità a geometria variabile nella ripartizione dei beni sociali che è in grado di tener conto delle reali differenze tra membri della comunità politica senza d'altra parte consentire ai potenti di abusare della loro forza. Questo concetto di equità "proporzionale" è poi esteso da Aristotele anche in ambiti rispetto ai quali sembrerebbe estraneo, come quello degli scambi commerciali e quello della "giustizia correttiva", cioè dell'erogazione di pene per delitti che ledono gli equilibri della comunità.

$$
* * *
$$

ABSTRACT. - In the book V of Ethica nicomachea, in the context of the discourse on "distributive justice" (a subsystem of "justice in general" or "political justice", essentially consisting in the respect for the law), Aristotle addresses the question of the fair distribution of social assets such as power functions, prestige positions, public wealth The democratic deception of a perfectly egalitarian distribution must be avoided as it does not correspond to the real social hierarchies of class and merit, and also to the greed (pleonexia) that distinguishes the oligarchic regimes: both classlessness and greed are seeds of social conflicts. Aristotle therefore proposes a concept of "proportional" equity, a kind of fairness with variable geometry in the distribution of social goods, which is able to take into account the real differences among members of the political community, while not allowing the powerful to abuse their power. Aristotle then extends this concept of "proportional" equity to apparently strange areas, such as trade and "corrective justice", namely the provision of penalties for crimes affecting the harmony of the community.

(*) Istituto Lombardo Accademia di Scienze e Lettere; Università degli Studi di Pavia, Italia. E-mail: mario.vegetti@icloud.com 
1. La lingua greca non dispone di un lessico dell'equità distinto da quello dell'eguaglianza ${ }^{1}$, a differenza delle coppie latine aequitas/aequus, aequalitas/aequalis.

Nella trattazione della giustizia condotta nel libro V dell'Etica nicomachea, Aristotele è perciò costretto a un difficile lavoro terminologico e concettuale per identificare uno spazio proprio alla nozione di "equità", che risulta particolarmente rilevante, come vedremo, nell'ambito della giustizia detta "distributiva".

Aristotele dunque specializza in questo senso l'aggettivo neutro sostantivato to ison, che presenta tuttavia due difficoltà. Da un lato, il suo significato primario è quello matematico di "uguale"; dall'altro, è connesso alla famiglia lessicale dell'eguaglianza (isotes, isonomia, isegoria) che risulta fin troppo carica di valenze politiche ${ }^{2}$. Ma la maestria

1 Si rende abitualmente con "equità" il termine epieikeia che compare nel capitolo V 14 dell'Etica nicomachea e nella Retorica (I 13.1374a-b) per indicare la necessità di "adeguare" la generalità delle leggi ai singoli casi particolari. Ciò che è epieikes rappresenta una correzione (epanorthoma) del giusto legale (EN V 14.1137b13), resa necessaria dall'universalità della legge che può risultare lacunosa nei casi singoli. Epieikeia varrà dunque "flessibilità" o "opportunità". L'uomo epieikes (dove si intende il buon giudice) tenderà dunque a non essere akribodikaios (rigoroso, inflessibile) ma elattotikòs (indulgente) (cfr. già in questo senso Platone, Leggi VI 757e). Si tratta di una problematica relativa all'amministrazione della legge che non rientra nella sfera del discorso etico-politico che vorrei svolgere qui. E' però vero che il problema dell'epieikeia, resa con aequitas, avrebbe occupato un ruolo centrale nella discussione giuridica in età moderna. Il miglior saggio filosofico sul tema è quello di J. Brunschwig, Rule and exception: on the Aristotelian Theory of Equity, in M. Frede- G. Striker (eds.), Rationality in Greek Thought, Oxford, Clarendon, 1996, 115-55. Più in generale, epieikeia ed epieikès indicano nell'Etica un tipo umano "adeguato" alle richieste sociali, perciò "dabbene", "valente", oggetto di "moral or social approval" (Brunschwig, 119). Sul rapporto tra il valore tradizionale del termine ("opportuno, appropriato alle circostanze") e quello aristotelico si vedano i saggi di F. Piazza (Dire e fare la cosa giusta. L'epieikeia in Aristotele, in C. Rossitto, ed., Studies on Aristotle and the Aristotelian Tradition, Edizioni di storia della tradizione aristotelica, Lecce 2011, pp. 159-76; La virtù di Emione. Riflessioni sull'epieikeia greca, Aevum antiquum, 2012), con le osservazioni di M. Bonazzi, La virtù di Creonte: qualche osservazione sul rapporto tra epieikeia e dike, Aevum antiquum (2009), 37-40.

2 I traduttori rendono di solito to ison con "uguale" in tutte le sue occorrenze, ciò che rende più difficile la comprensione dell'argomentazione aristotelica. Natali propone di differenziare il significato traducendo secondo i casi "eguale" e "onesto" (questo secondo aggettivo è più vicino al significato di "equo", ma si riferisce prevalentemente 
con cui il filosofo si muove all'interno di questa difficoltà lessicale produrrà risultati interessanti, e tutt'altro che neutrali, nel quadro concettuale del problema della giustizia.

Il problema dell'equità non si pone, almeno inizialmente, per quello che Aristotele definisce "il giusto in generale", cioè il giusto che riguarda le regole che garantiscono una soddisfacente convivenza nella comunità politica: il giusto politico coincide con l'osservanza della legge, anche se questa sommaria identificazione apre un altro problema, quello dell'esistenza di leggi ingiuste, che Aristotele discute con la sua abituale circospezione, e che non ci interessa direttamente in questa sede ${ }^{3}$.

$\mathrm{Ci}$ sono però due sfere particolari della giustizia, che riguardano sottosistemi di relazioni presenti all'interno della comunità politica. Il primo di essi consiste nella sfera della distribuzione o ripartizione di beni materiali o simbolici. Nell'ambito pubblico, si tratta della ripartizione (dianomé) di "onori" (timé), ricchezze (chremata) e di altri beni distribuibili (meristà) fra i cittadini membri della comunità (V 5.1130b30-33); nell'ambito privato si tratta degli scambi commerciali. Il tipo di "giusto" relativo a questo doppio ambito viene definito da Aristotele "distributivo" (dianemetikòn dikaion).

Il secondo sottosistema consiste nella sfera del diritto penale o civile; il tipo di "giusto" ad esso relativo è definito "correttivo" (diorthotikòn dikaion). Benché la questione dell'equità si ponga in tutti questi ambiti, essa risulta particolarmente rilevante, e concettualmente impegnativa, soprattutto nel primo, quello della ripartizione dei beni sociali fra i membri della comunità politica, da cui tra l'altro derivano notevoli ricadute, formali e sostanziali, anche sulla dimensione politica.

2. Le premesse dell'argomentazione di Aristotele vanno ricostruite con attenzione. Il giusto, egli scrive con una formula sintetica che riprende la sua tesi generale, coincide con il "legittimo", nomimon, e

all'ambito degli scambi commerciali). Fermani interpreta to ison come "equo", insieme con epieikeia.

3 Nella Nicomachea (V 2.1129b25) Aristotele si limita a considerare il caso di leggi malfatte o "affrettate”, ma nella Politica (III 4) verrà ampiamente discussa l'esistenza di regimi "deviati” (tirannide, oligarchia, democrazia) con la relativa legislazione, cui il "buon cittadino" dovrà conformarsi, ciò che lo distanzia dalla condizione dell'uomo moralmente "buono". 
anche con l'ison, dove l'aggettivo non può valere "eguale" ma appunto "equo", come risulta dalla parallela definizione dell'ingiustizia. Essa coincide con l' "illegittimo" (paranomon), con l' "iniquo" (anison) (V $5.11308-10$ ), che a sua volta può venire identificato con la pretesa arbitraria di ottenere una parte maggiore dell'equo e del lecito, cioè con quella pulsione della pleonexia, prevaricazione (V 2.1129a32-b2), che costituisce un aspetto centrale della discussione antropologica greca fra $\mathrm{V}$ e IV secolo.

In questo rapporto dell'equità con la legittimità, e tramite essa con la legge, è da vedere un primo nesso, di carattere formale, tra la sfera dell'equità e quella della politica, di cui la legge è la norma di giustizia.

Ma l'analisi aristotelica farà emergere un rapporto più sostanziale tra le due sfere.

L'ingiustizia - come ogni condotta che presenti una dimensione quantitativa e misurabile - consiste nel "troppo" (lo scopo della pleonexia) e simmetricamente nel "troppo poco" (ad ogni appropriazione eccessiva corrisponde una deprivazione iniqua). Fra questi due estremi, com'è proprio dell'etica aristotelica, sta un "giusto mezzo", un meson, che è rappresentato dall'ison, "eguale" nel senso di pareggiare gli opposti eccessi, in cui consiste appunto il "giusto" (V 6.1131a9-5). Ma Aristotele è subito attento a controllare gli effetti di questa medietà apparentemente egualitaria, e a ristabilire il valore di ison come "equo" anziché come uguale.

3. Nel campo della ripartizione dei beni sociali, il "giusto" comporta sempre, nella sua figura essenziale, quattro termini: due sono le persone coinvolte, e gli altri due sono quote di beni da distribuire. L'eguaglianza che è pertinente a questo ambito della giustizia non è quella aritmetica $(1=1)$ ma quella della proporzione geometrica. Viene dunque costruito questo ragionamento (Etica nicomachea V 6-7):

Siano

A, B persone; C, D quote di beni sociali ripartibili

Equità proporzionale:

$$
\begin{aligned}
& A: B=C: D \\
& A: C=B: D \\
& (A+C):(B+D)=A: B
\end{aligned}
$$


Solo nel caso $A=B$ [democrazia $]$

$(1+1):(1+1)=1: 1$

Se $A \neq B$ (es. $A=2 x)$

$(2+2):(1+1)=2: 1$

Come si vede, quello della democrazia è l'unico caso in cui l'eguaglianza numerica coincide con l'equità proporzionale. Si tratta di un effetto dell'illusione - o piuttosto dell'inganno - della democrazia, che Platone aveva già sinteticamente indicato nella Repubblica: «questo anarchico regime distribuisce una sorta di eguaglianza (isoteta) parimenti agli uguali (isoi) e ai diseguali (anisoi)» (VIII 558c). Che cosa significa questo uso assoluto di concetti relativi come quelli di uguale e diseguale? Platone intende dire che la democrazia tende a egualizzare in ambito politico, non solo nel senso formale dell'eguaglianza di fronte alla legge, ma in quello sostanziale della distribuzione di beni sociali come prestigio, cariche pubbliche, ruoli di potere, remunerazioni, le differenze sociali di censo, di ceto, di cultura che non vengono cancellate ma in qualche modo nascoste dalla isotes democratica ${ }^{4}$.

Aristotele riprende, amplia e chiarisce la posizione platonica. Il giusto, egli scrive nella Politica, sembra essere l'eguale (to ison), e in effetti lo è, ma non verso tutti, bensì soltanto per gli eguali (tois isois). Viceversa, il diseguale (to anison) sembra essere giusto, e lo è, ma non per tutti bensì per chi è diseguale (III 9.1280a7-8). Qui si produce l'inganno democratico. I democratici partono dalla constatazione di un

4 Il testo platonico più vicino alla riflessione aristotelica è però quello di Leggi VI 757a-758a. Con una sorta di gioco di parole, Platone scrive che «parti uguali (ta isa) tra diseguali diventerebbero diseguali (anisa), se mancasse la misura». In che cosa consista questa misura è precisato poco più avanti: l'eguaglianza migliore, conforme al giudizio di Zeus, «impartisce di più al più grande, di meno al più piccolo, dando a ciascuno dei due in giusta misura secondo la loro natura». Per evitare sedizioni, in qualche caso è consigliabile adottare la forma paritaria dell'eguaglianza, quella che nella democrazia ateniese si esprime per eccellenza nell'assegnazione delle cariche per sorteggio. «Per evitare il malcontento delle masse è necessario valersi dell'eguaglianza dovuta a sorteggio, anche allora invocando nelle preghiere la divinità e la buona fortuna di indirizzare la sorte verso la maggiore giustizia. Così ci si deve necessariamente valere delle due forme di eguaglianza, ma in modo da servirsi il meno possibile di quella delle due che necessita della sorte». 
dato giuridico, l'eguaglianza di tutti i cittadini per la comune condizione di libertà (cioè di non schiavitù), e chiedono su questa base che venga riconosciuta l'uguaglianza in assoluto (holôs, haplôs), da cui deriverebbe il diritto a ricevere una quota paritaria nella distribuzione dei beni sociali (pantôn tôn isôn metechein) (Pol. III 9.1280a23-25, V 1.1301a26-39).

Simile, anche se meglio fondata, la pretesa degli oligarchi. In ragione del fatto che essi sono "diseguali" per il possesso di ricchezza, pretendono di avere una parte eccessiva e iniqua nella ripartizione (pleonektein) (un'aspirazione, nota Aristotele, che a volte può risultare giusta, altre volte ingiusta, probabilmente se rispetta o meno l'analogia di proporzione, Pol. V 2.1302a26-27).

E' fondamentalmente da questo dissenso sull'estensione dell'eguaglianza, e sulla conseguente valutazione dell'equità, che nascono secondo Aristotele conflitti e rivendicazioni nella ripartizione dei beni sociali (Eth. nicom. V 6.1131a23-24), che possono degenerare fino alla guerra civile, la stasis (Pol. V 2.1302a29-32: «gli inferiori si ribellano per ottenere l'eguaglianza, e coloro che sono uguali si ribellano per ottenere dei privilegi. Coloro che si ribellano lo fanno per ottenere guadagni (kerdos) e onori»).

Occorre dunque individuare un' axia, un criterio di valore o di merito sul quale fondare la giusta proporzione nella distribuzione fra soggetti diversi delle quote dei beni sociali (Eth. nicom. V 6.1131a2531). Per i democratici, come si è visto, questo criterio consiste nella condizione giuridica di libertà. Ma si tratta di un criterio erroneo, perché trasforma necessariamente l'equità proporzionale in eguaglianza (cioè assegna l'eguale a chi in realtà non è eguale). Per gli oligarchici, il criterio va individuato nella ricchezza e nella nobiltà: una richiesta parzialmente sensata, ma pericolosa perché suscita quel sospetto di avidità dei ricchi (pleonexia) che è foriero di stasis.

Ci sono infine gli "aristocratici" che rivendicano il criterio della "virtù" (areté). Questa posizione suscita qualche dubbio. Un'aristocrazia slegata da condizioni di censo e fondata sulla sola areté - dunque sulla qualità morale e intellettuale - costituisce un'eco indubbia del "potere virtuoso" di memoria platonica, ma poco in sintonia con il pensiero aristotelico. Ancor meno lo sarebbe l'idea di un'aristocrazia morale dei poveri e dei plebei, con la sua impronta cinica. A che cosa dunque può pensare Aristotele?

Bisogna probabilmente richiamare il valore prestazionale, oltre 
che morale, della parola areté, ancora ben presente nella semantica aristotelica. L'aristocrazia della virtù sarà dunque composta da coloro che sono in grado di fornire prestazioni eccellenti alla comunità politica, in pace come in guerra in aggiunta alla ricchezza e alla nobiltà, e anche, eccezionalmente, in sostituzione di esse. Si tratta dei cittadini che Aristotele chiama spoudaioi, seri, efficaci e virtuosi, in cui Aristotele riconosce il canone ostensibile del comportamento morale (cfr. ad es. Eth. nicom. III 6.1113 a32 ss., IX $4.1166 a 12$ s.). E' significativo che Aristotele nomini, come tipi emblematici di questa figura di cittadino, «Pericle e i suoi simili» (Eth. nicom. VI 5.1140b8). L'aristocrazia virtuosa di Aristotele non sarà dunque come quella intellettuale di Platone, perché si compone dei cittadini eminenti già attivi e riconoscibili sulla scena della comunità politica, e tanto meno come quella cinica, perché - come il caso di Pericle dimostra - essa è tutt' altro che povera e plebea. Si tratta insomma, noi potremmo dire, di un'aristocrazia meritocratica, e ai suoi membri tocca quell'assegnazione proporzionale dei beni sociali in cui la diseguaglianza delle quote rispetta equamente la differenza di valore individuale, e perciò non dovrebbe fomentare dissensi e rivolte.

Viene così configurata un'immagine del potere politico-sociale sostanzialmente non diversa da quella tradizionale, ma rilegittimata dall'analisi su giustizia ed equità. «Il governante [l'uomo di potere, archon] è custode del giusto, e se lo è del giusto lo è anche dell'equo. Se dunque è giusto, sembra che non debba avere nulla in più degli altri: non si attribuirà infatti una quota maggiore di beni in generale, se non nella misura in cui ciò rispetti la proporzionalità rispetto al suo stato...si deve dunque dargli una certa ricompensa, cioè onori e vantaggi; chi non se ne accontenta, diventa tiranno» (Eth. nicom. V 10.1134b1-9).

4. Negli altri sottosistemi della giustizia, quello che riguarda gli scambi di beni fra privati, e quello che riguarda la giustizia penale o "correttiva” vige in linea di principio la norma dell'eguaglianza numerica e non proporzionale, sicché essa dovrebbe coincidere con l'equità.

$\mathrm{Ma}$ Aristotele non è del tutto conseguente con questa premessa. Prendiamo il caso degli scambi. Sia A un architetto, B la casa che costruisce, $\mathrm{C}$ un calzolaio, D le scarpe che produce. Per ottenere uno scambio equo occorre rendere omogeneo il valore delle scarpe con quello della casa, e a questo scopo, nota Aristotele, è stato introdotto l'equivalente generale costituito dalla moneta. Se dunque una scarpa 
vale 1 dracma, e una casa 1000 dracme, per acquistare la casa occorrerà l'equivalente di 1000 scarpe. Ma come determinare il "valore" di case e scarpe? Aristotele, naturalmente, non vuole o piuttosto non può vedere la funzione valorizzante del lavoro (tanto meno in una società in cui la produzione è basata sul lavoro servile, e in cui dunque non esiste, o è marginale, un mercato della forza-lavoro). Di fronte a questa domanda, invece che una qualsiasi teoria dei prezzi, ricompare l'equità come proporzionalità fra i soggetti sociali dello scambio: «è necessario che, come un architetto sta a un calzolaio, così questa precisa quantità di scarpe stia a una casa; se non si dà ciò, cioè se i beni non saranno in qualche modo egualizzati, non avremo né scambio né comunità» (Eth. nicom. V 8.1133 a22-25). La ratio della scambiabilità di merci su una base di equità sta dunque nel "valore" non economico ma sociale riferibile allo status dei loro produttori, esattamente come quella della ripartizione dei beni sociali. Arcaismo economico e deformazione ideologica, forse, ma anche un approccio che riflette la situazione storica di una sfera economica che, come ha detto Polanyi, era ancora embedded nel sistema dei rapporti politico-sociali (la sua iniziale autonomizzazione sarebbe stata compresa, e criticata sotto il nome di "crematistica innaturale", da Aristotele nel I libro della Politica).

Simile, ma per certi aspetti più sorprendente, l'applicazione dell'equità proporzionale all'ambito della giustizia "correttiva". Qui l'approccio aristotelico è dichiaratamente aritmetico. La pena è concepita secondo il modello del risarcimento: se $P$ ha ingiustamente tolto $x$ a $Q$, il giudice dovrà togliere $x$ a $P$ e riassegnarlo a $Q$ : si tratta dunque di ristabilire l'eguaglianza; aggiunge Aristotele che «non fa nessuna differenza se sia stato un uomo dabbene (epieikes) ${ }^{5}$ a derubare un uomo dappoco, o sia stato un uomo dappoco a derubare un uomo dabbene, né se a commettere adulterio sia stato l'uomo dabbene o quello dappoco; la legge riguarda solo la differenza prodotta dal danno, e tratta le due parti come uguali» (Et. nicom. V 7.1132a2-5). A parte la difficoltà di capire come il modello lineare del risarcimento sia applicabile in casi diversi dall'appropriazione indebita, per esempio nell'adulterio o nell'omicidio, questa rigorosa eguaglianza di fronte alla legge non regge in Aristotele, e nel capitolo seguente il principio dell'equità proporzionale rientra decisamente sulla scena. Esso si presenta ora come una versione

5 Qui l'aggettivo compare nel più consueto significato morale-sociologico. 
del principio di giustizia già sostenuto dai Pitagorici, il "contraccambio" o reciprocità (antipeponthòs). Questo principio, dice Aristotele, non può venire applicato secondo una reciprocità lineare: «se chi detiene una carica ha percosso qualcuno, non deve essere percosso a sua volta, mentre se uno ha percosso un governante deve ricevere non solo delle percosse ma anche venire punito». Così concepito, il principio di reciprocità ha un valore importante: «il contraccambio secondo la proporzione e non secondo l'eguaglianza (katà analogian kai mê kat' isoteta) è fonte di connessione, dato che la città sussiste grazie al contraccambio proporzionale» (V 8.1132b29-1133a1).

La lettura del V libro della Nicomachea sulle tracce dello sfuggente concetto di equità consente, alla fine, di mettere in luce un non insignificante esperimento teorico aristotelico: $i$ lineamenti di una geometria dei rapporti sociali che da un lato rispetta i dislivelli propri di una società di status, evitando la pericolosa tendenza egualitaria della democrazia, dall'altro però mira a regolarne gli effetti combattendo l'opposta tendenza alla prevaricazione e alla sopraffazione, perché tanto l'eguaglianza quanto la pleonexia sono foriere, secondo Aristotele, del pericolo di minare la coesione della comunità politica e di esporla al conflitto civile, all'incombente ben nota minaccia della stasis ${ }^{6}$.

E' invece naturalmente estranea all'orizzonte aristotelico l'idea di usare il dispositivo dell'equità proporzionale in senso opposto, cioè ripartendo i beni sociali in modo da avvantaggiare chi ha meno, così da ridurre la diseguaglianza sociale e di riavvicinare equità ed eguaglianza.

6 Si vedano sul problema le osservazioni equilibrate, anche se forse troppo indulgenti con Aristotele, di E. Berti, La giustizia come proporzione, in A. Ferrara - V. Gessa Kurotschka - S. Maffettone (a cura di), Etica individuale e giustizia, Napoli, Liguori, 2000, 57-68 (anche in relazione a A. McIntyre, Giustizia e razionalità, Milano, tr. it. Anabasi, 1995). 